Tian, W., Han, X., Zuo, W., Wang, Q., Fu, Y. and Jin, M., 2019. An Optimization Platform Based on Coupled Indoor Environment and HVAC Simulation and Its Application in Optimal Thermostat Placement. Energy and Buildings. https://doi.org/10.1016/j.enbuild.2019.07.002

An Optimization Platform Based on Coupled Indoor Environment and HVAC Simulation and Its Application in Optimal Thermostat Placement

Wei Tian ${ }^{a+}$, Xu Han ${ }^{b}$, Wangda Zuo ${ }^{b, c^{*}}$, Qiujian Wang ${ }^{d}$, Yangyang $\mathrm{Fu}^{\mathrm{b}}$, Mingang Jin ${ }^{\mathrm{e}}$

${ }^{a}$ Department of Civil, Architectural and Environmental Engineering, University of Miami, 1251 Memorial Drive, Coral Gables, FL 33146, U.S.A.

${ }^{b}$ Department of Civil, Environmental and Architectural Engineering, University of Colorado Boulder, UCB 428, Boulder, CO 80309, U.S.A.

${ }^{c}$ National Renewable Energy Laboratory, Golden, CO 80401, USA

${ }^{d}$ College of Mechanical and Energy Engineering, Tongji University, Shanghai, China

${ }^{e}$ Emerson Automation Solutions, Houston, TX 77041, U.S.A.

${ }^{+}$Current Affiliation: Schneider Electric, 800 Federal Street, Andover, MA 01810, U.S.A.

*Corresponding Author information: Email: Wangda.Zuo@colorado.edu; Phone: 303-492-4333; Address: ECCE 247, UCB 428, Boulder, CO 80309 


\title{
An Optimization Platform Based on Coupled Indoor Environment and HVAC Simulation and Its Application in Optimal Thermostat Placement
}

\begin{abstract}
Model-based optimization can help improve the indoor thermal comfort and energy efficiency of Heating, Ventilation and Air Conditioning (HVAC) systems. The models used in previous optimization studies either omit the dynamic interaction between indoor airflow and HVAC or are too slow for model-based optimization. To address this limitation, we propose an optimization methodology using coupled simulation of the airflow and HVAC that captures the dynamics of both systems. We implement an optimization platform using the coupled models of a coarse grid Fast Fluid Dynamics model for indoor airflow and Modelica models for HVAC which is linked to the GenOpt optimization engine. Then, we demonstrate the new optimization platform by studying the optimal thermostat placement in a typical office room with a VAV terminal box in the design phase. After validating the model, we perform an optimization study, in which the VAV terminal box is dynamically controlled, and find that our optimization platform can determine the optimal location of thermostat to achieve either best thermal comfort or least energy consumption, or the combined. Finally, the time cost for performing such optimization study is about 6.2 hours, which is acceptable in the design phase.
\end{abstract}

Keywords: FFD, Modelica, coupled simulation, optimization, thermostat placement

\section{Introduction}

HVAC systems with non-uniform airflow and temperature distributions are widely adopted in the building design to achieve good thermal comfort and energy efficiency. Typical examples include displacement ventilation (Yuan et al. 1999), data centers with raised-floor plenum architecture (VanGilder et al. 2018), and ventilation systems with atrium (Chen 2009). As suggested by Tian, Han, et al. (2018), coupled simulation models between Building Energy 
Simulation (BES) and Computational Fluid Dynamics (CFD) can be used to study the design and operation of such systems. To find the optimal design and operation conditions, model-based optimization can be performed using the coupled simulation models.

Plenty of researchers have presented the optimization of HVAC system operation using BES or optimal indoor airflow distribution using CFD. Nguyen et al. (2014) reviewed the optimization method using BES to study the building performance. Delgarm et al. (2016) performed multiobjective optimization using EnergyPlus simulation and particle swarm optimization (PSO) scheme that can improve the energy performance of the design. The simulation-based optimization methodology and its variants (using difference simulator and/or optimizations schemes) has been applied to the design of energy efficient buildings in different forms (general buildings, data centers, etc.), and promising results in terms of whole-year-energy-consumption reductions were presented. Similarly, the optimization methodology based on single simulator has been applied to study the control performance of building energy systems in a dynamic fashion (Tian, Zhang, et al. 2018; Chen et al. 2018; Fu et al. 2019; Afram et al. 2017; D'Agostino and Parker 2018; Han et al. 2013). For example, Huang et al. (2016) and Huang et al. (2017) applied the optimization based on the Modelica representation of the HVAC system to find the optimal set point of condensing water temperature and chiller staging control. Wang et al. (2017) applied optimization based on the Modelica representation of duct network to find the optimal opening of the valves. On the other hand, Liu et al. (2015) gave an overview on utilizing the optimization based on CFD to carry out inverse design. The adjoint method was used to optimize the air supply location, size and parameter for enclosed spaces, such as aircraft cabin (Liu et al. 2017). Lee (2007) and Xue et al. (2013) used genetic algorithm based on CFD simulations to optimize the flow control conditions for the indoor climate. The abovementioned researches have shown that the simulation-based optimization can 
reduce energy consumption of HVAC systems and achieve desired thermal environment conditions. However, none of the above research considered the complex and dynamic interactions between HVAC and indoor environment, which may lead to incorrect simulation results and a potentially more serious consequence-failure of the system. In buildings, sub-domains can be interconnected from the physical perspective. For example, in a feedback loop control of air handler and thermal indoor environment, the temperature at a specific spot in the environment is extracted and sent to the control module, which then modulates the air handler to adjust the cooling; The states of the air handler, especially the temperature and velocity at the exhaust, consequently determines the airflow and temperature distributions in the space. The interactive entangling of the two domains-mainly thermal environment and cooling system should be addressed in a proper way such that the important "coupling" will not be over-simplified (using multizone model to model a non-uniform temperature distribution) or omitted (assume the temperature at the specific spot same as the setpoint). Moreover, with increased attention on the design of the control system, the essence of being dynamic of the control process should be properly addressed. For example, Wetter (2009b) noted the inability to resolve the non-linear dynamic behavior of building energy system and its control system can lead to equipment shortcycling. Likewise, Nassif et al. (2008) noted the importance of dynamic models of HVAC systems in designing energy management control system. One can refer to literatures for more information on dynamic modeling of HVAC systems (Li et al. 2014).

It is important to note that the optimization based on coupled simulation has been carried out in a few studies, which are typically not in the domain of interacting HVAC with indoor thermal environment. For example, with resilience and energy efficiency becoming a hot research topic, there is emerging of researches on optimizing the integration of renewable/distributed energy 
sources. For example, O'Shaughnessy et al. (2018) developed Solar Plus to increase the economic befit by performing optimization based on coupled simulations of PV panels, batteries under various electricity-rate structures. Ma and Xia (2017) presented a simulation-based optimization to control the ground source heat pump system by considering the interaction between heat pump, water pump, and ground heat exchanger. Yang et al. (2017) analyzed the performance and control strategy of a combined cooling, heating and power system in a hotel, by modeling the interaction of the system with solar thermal energy and compressed air energy storage. Han et al. (2017) studied the energy performance of the timber-glass buildings by performing optimization based on coupled simulation of daylighting using Radiance and building energy performance using EnergyPlus. Similarly, in the domain of regional heating, Pan et al. (2017) developed a feasible region method to improve the efficiency of integrated heat and electricity system by considering the interaction of two models at the same time.

It is noteworthy that there is a paper attempting to perform optimization based on coupled simulation of HVAC systems and indoor thermal environment. Du et al. (2015) performed an optimization study to find the temperature sensor placement using coupled simulation between TRNSYS and CFD. However, there are few limitations to this study. First, the CFD simulation was performed in steady-state. Although it is believed to reduce the computational cost (as transient CFD simulation is costly), the steady-state CFD results may adversely affect the control simulation of the HVAC system. Consequently, a real dynamic interaction of HVAC systems and indoor environment was not considered. Second, even with steady-state simulations, the computational speed is still too slow to perform optimization over a large search domain. As a result, only a handful of discrete locations were picked, among which the optimal placement was determined. 
In this paper, we first discuss the methodology and implementation of the proposed the optimization using dynamically coupled simulation of indoor environment and HVAC systems. We then identify an office room with displacement ventilation, in which we demonstrate how to use the optimization platform to determine the thermostat location to achieve optimal thermal comfort, energy efficiency, or both. The paper is structured as follows: section 2 discusses the methodology and detailed implementation of the optimization platform; section 3 discusses the evaluation of the coupled simulation model; section 4 introduces the optimization studies; and section 5 gives the conclusion and future work.

\section{Methodology and Implementation}

\subsection{Methodology of the coupled simulation platform}

This paper proposes an optimization platform using the dynamically coupled simulation of HVAC system and non-uniform indoor environment. The platform can be harnessed to improve the control and energy performance of cooling system in various applications. As a prominent case, data center, which consumes about $2 \%$ of the electricity in the U.S.(Shehabi et al. 2016), can benefit from this platform to improve the energy efficiency and reduce the green gas emission. The whitespace in the data center used to house the racks is typically characterized with non-uniform temperature distribution. The temperatures at designated locations are sampled to control the computer room air conditioner. The optimization scheme proposed in this paper can be used to improve cooling energy efficiency while attaining the required thermal environment to run the IT equipment safely. To accelerate the computational speed of the coupled simulation, our proposed optimization platform employs a coupled simulation model with Modelica and Fast Fluid Dynamics (FFD) (Zuo et al. 2016). Modelica is an equation-based and object-oriented language

for dynamic simulation. A Modelica Buildings library (Wetter et al. 2014) was developed to 
facilitate building system modeling. FFD, which simulates airflow and temperature distribution in transient state, is about 50 times faster than its counterpart CFD (Zuo and Chen 2009) and even faster when adopting more advanced semi-Lagrangian algorithm (Mortezazadeh and Wang 2017). Parallelization of FFD to run on multi-core device can further increase the speed up to 1000 times (Tian, Sevilla, and Zuo 2017; Zuo and Chen 2010). In addition, to further accelerate the simulation, we implemented a coarse grid solver in FFD, which was reported to be able to accelerate the FFD simulation for 5-50 times (Jin et al. 2015). The integration of all these techniques makes the coupled-simulation-based optimization possible by dramatically reducing the computational cost of the coupled simulation, in which the airflow simulation is the bottleneck of the couple simulation speed. It is noteworthy that reduced-order models (Tian, Sevilla, et al. 2018), though computationally fast, are mostly for steady-state predictions, and therefore are not good for dynamic simulations.

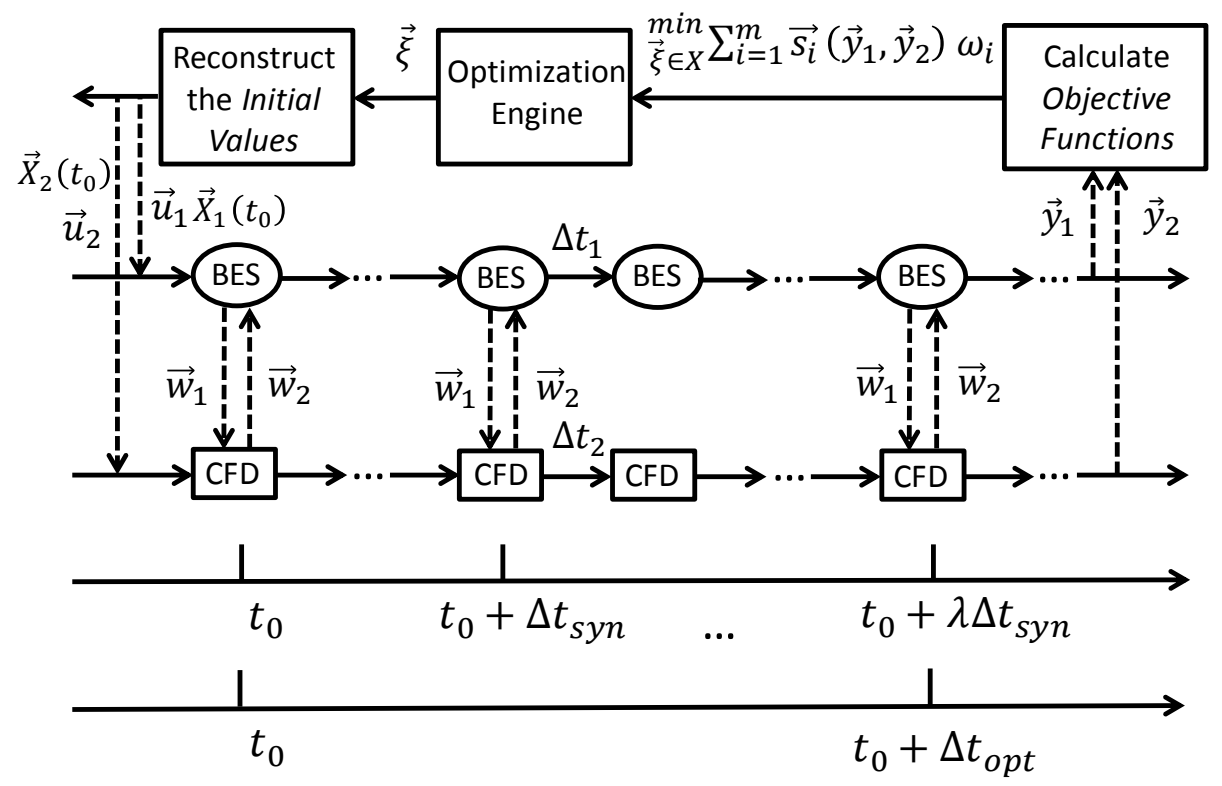

Figure 1 Optimization Methodology Based on Coupled CFD-BES Simulation

Figure 1 shows the framework of the optimization platform using the dynamic coupling model 
between BES for HVAC and CFD for non-uniform indoor environment. The whole optimization process is divided into multiple optimization steps, in which optimization is performed to find the optimal solution. The optimal together with the old states from the last optimization step are used to initialize the next optimization step. The number of the optimization steps are determined by the users based on their interpretation of physics to be optimized. It is noteworthy that a tradeoff between optimization accuracy and speed is associated with the optimization time step.

At the heart of this optimization platform is the coupled simulation between BES and CFD. The coupled simulation employs a quasi-dynamic coupling scheme, and was implemented based on a customized interface, in which the Modelica model exchanges data with FFD dynamic link library through external "C" functions. Note that there are various data synchronization schemes and software implementation architectures available to couple the BES with CFD. For example, quasi-dynamic scheme requires data exchange once at a data synchronization time point while fully-dynamic scheme requires several iterations of data exchanges until both BES and CFD reach converged solution. Consequently, quasi-dynamic scheme is relatively computationally faster and more stable while fully-dynamic scheme is tending to be able to generate more accurate results. Other than customized interface to enable data exchange and control of the coupled simulation, middleware interface and standard interface can also be utilized. Unlike the master-slave mode used in this implementation, middleware, such as building control virtual test bed (Wetter 2011), can play the role of controlling the coupled simulation and provide better maintainability and extendibility when compared to the customized interface. The standard interface, which enables two programs being connected directly, is straightforward and easy-to-deploy. Take functional mockup interface as an example, tools that are compatible with the functional mockup interface standard can export models as functional mockup units, which can be then coupled (it can be as 
easy as plug-and-play) and simulated in co-simulation environment such as pyFMI (Blochwitz et al. 2011). One can refer to the literature review paper (Tian, Han, et al. 2018) for detailed explanation. Assume we have the following general equations to describe the behaviours of BES and CFD for the optimization step $\left(t_{0}, t_{0}+\Delta t_{\text {opt }}\right)$ :

$$
\begin{gathered}
\dot{\vec{x}}_{1}=\vec{f}_{1}\left(\vec{x}_{1}, \vec{u}_{1}, \vec{w}_{2}\right), \vec{x}_{1}\left(t_{0}\right)=\hat{x}_{1}\left(t_{0}\right) \\
\vec{u}_{1}=\overrightarrow{\psi_{1}}\left(\hat{u}_{1}\left(t_{0}\right), \vec{\xi}\right) \\
\vec{y}_{1}=\overrightarrow{g_{1}}\left(\vec{x}_{1}, \vec{u}_{1}, \vec{w}_{2}\right) \\
\vec{w}_{1}=\overrightarrow{h_{1}}\left(\vec{x}_{1}, \vec{u}_{1}, \vec{w}_{2}\right) \\
\vec{x}_{2}=\overrightarrow{f_{2}}\left(\vec{x}_{2}, \vec{u}_{2}, \vec{w}_{1}\right), \vec{x}_{2}\left(t_{0}\right)=\hat{x}_{2}\left(t_{0}\right) \\
\vec{u}_{2}=\overrightarrow{\psi_{2}}\left(\hat{u}_{2}\left(t_{0}\right), \vec{\xi}\right) \\
\vec{y}_{2}=\overrightarrow{g_{2}}\left(\vec{x}_{2}, \vec{u}_{2}, \vec{w}_{1}\right) \\
\vec{w}_{2}=\overrightarrow{h_{2}}\left(\vec{x}_{2}, \vec{u}_{2}, \vec{w}_{1}\right)
\end{gathered}
$$

where the subscript in the names of function and variable names indicate the program ("1" for BES; "2" for CFD). $\vec{x}_{1}$ and $\vec{x}_{2}$ are vectors of state variables. $\dot{\vec{x}}_{1}$ and $\overrightarrow{\vec{x}}_{2}$ are derivatives of $\vec{x}_{1}$ and $\vec{x}_{2}$ with respect to time. $\vec{y}_{1}$ and $\vec{y}_{2}$ are vectors of outputs. $\vec{u}_{1}$ and $\vec{u}_{2}$ are the inputs of BES and CFD, respectively. $\vec{w}_{1}$ and $\vec{w}_{2}$ are exchanged data between BES and CFD. The initial states of $\vec{x}_{1}$ and $\vec{x}_{2}$ the states $\left(\hat{x}_{1}\left(t_{0}\right), \hat{x}_{2}\left(t_{0}\right)\right)$ from last optimization step. The input vectors may be a function of the initial inputs (given prior to the optimization, i.e. $\hat{u}_{1}\left(t_{0}\right), \hat{u}_{2}\left(t_{0}\right)$ ) and optimized values $(\vec{\xi})$. We note that optimized values $(\vec{\xi})$ are the intermediate (stepwise) values of the input variables being optimized in the optimization process. When the optimization completes, the optimized value will be the optimal solution.

Even though above equations can be solved simultaneously (aggregated simulation or internal 
coupling), this methodology prefers the approach of external coupling (co-simulation). Within a data synchronization time step $\Delta t_{s y n}, \vec{w}_{1}$ and $\vec{w}_{2}$ are held constant when used as inputs. Theoretically, the synchronization time step $\Delta t_{s y n}$ should adapt to address the events in both simulations, so that the coupled simulation results are accurate. Here we note that events in the context of numerical simulation are referred to behaviours that can cause discontinuity of system response. Examples are turning on/off air conditioners, staging on/off chillers, etc. Without using proper smoothing techniques, these events can introduce step-change in the results and consequently bring challenges to the co-simulations in terms of controlling the co-simulation time step size. A typical trial-and-error approach, such as in functional mockup interface standard, is used to iteratively locate the event and then further adapt the co-simulation time step. This is particularly important when the numerical model is not properly smoothed (the response of the system is not always continuous), as using a constant co-simulation in such occasion could bring erroneous exchange data and instability to the co-simulation.

The optimization problem at the optimization step $\left(t_{0}, t_{0}+\Delta t_{\text {opt }}\right)$ can be formulated to find $\vec{\xi}$ such that:

$$
\min _{\vec{\xi} \in X} \sum_{i=1}^{m} \vec{s}_{l}\left(\vec{y}_{1}, \vec{y}_{2}\right) \vec{\omega}_{i}, \text { where } \sum_{i=1}^{m} \vec{\omega}_{i}=1
$$

where $\overrightarrow{s_{l}}$ is a vector of functions to map the outputs of the coupled simulation to optimization objective functions, and $\omega$ is the weight that converts a multi-objective into a single-objective optimization. $m$ is the number of objectives. $X$ is the searching domain for independent variables. The optimization process consists of following steps:

- Reconstruct the initial values: At time $t_{0}$, the initial conditions and inputs should be given before solving the set of equations from (1) to (8). Both BES and CFD should be able to read the states and inputs from the external sources. 
- Perform coupled simulations: Solving the set of equations from (1) to (8) using a cosimulation approach for $t_{0}$ to $t_{0}+\Delta t_{\text {opt }}$ to yield the outputs $\vec{y}_{1}$ and $\vec{y}_{2}$. These outputs can be transient or time-averaged.

- Calculate objective functions: Analytically evaluating the formula (9) can be difficult, if not impossible. To solve the optimization through a numerical approach, this step evaluates $\sum_{i=1}^{m} \vec{s}_{l}\left(\vec{y}_{1}, \vec{y}_{2}\right) \omega_{i}$ before calling optimization algorithms to find the optimal.

- Call optimization engine: Based on the value of current and possibly historical values of the objective functions (either local search or global search), the optimization engine updates the optimal $\vec{\xi}$ within the search domain $X$. The updated $\vec{\xi}$ will be used to further update the input vectors.

- Repeat above process until the optimization converges to optimal values of inputs for current optimization period from $t_{0}$ to $t_{0}+\Delta t_{\text {opt }}$.

- Repeat above process for next optimization period from $t_{0}+\Delta t_{\text {opt }}$ to $t_{0}+2 * \Delta t_{\text {opt }}$.

\subsection{Implementation of Optimization Platform}

While the optimization platform can be implemented in various ways, this paper chooses Modelica and Fast Fluid Dynamics (FFD) to carry out the coupled simulation and PSO algorithm in GenOpt (Wetter 2009a) as the optimization engine. Modelica, an equation-based and objectoriented language, is designed for dynamic simulation of the components and systems in various physical domains, i.e., component models or system models of HVAC. FFD is picked for airflow and thermal environment simulation thanks to its fast computational-speed. Previous research has demonstrated that the coupled simulation model between Modelica and FFD can provide a realistic environment to study the dynamic interaction of the stratified air distribution and HVAC system (Zuo et al. 2016). 


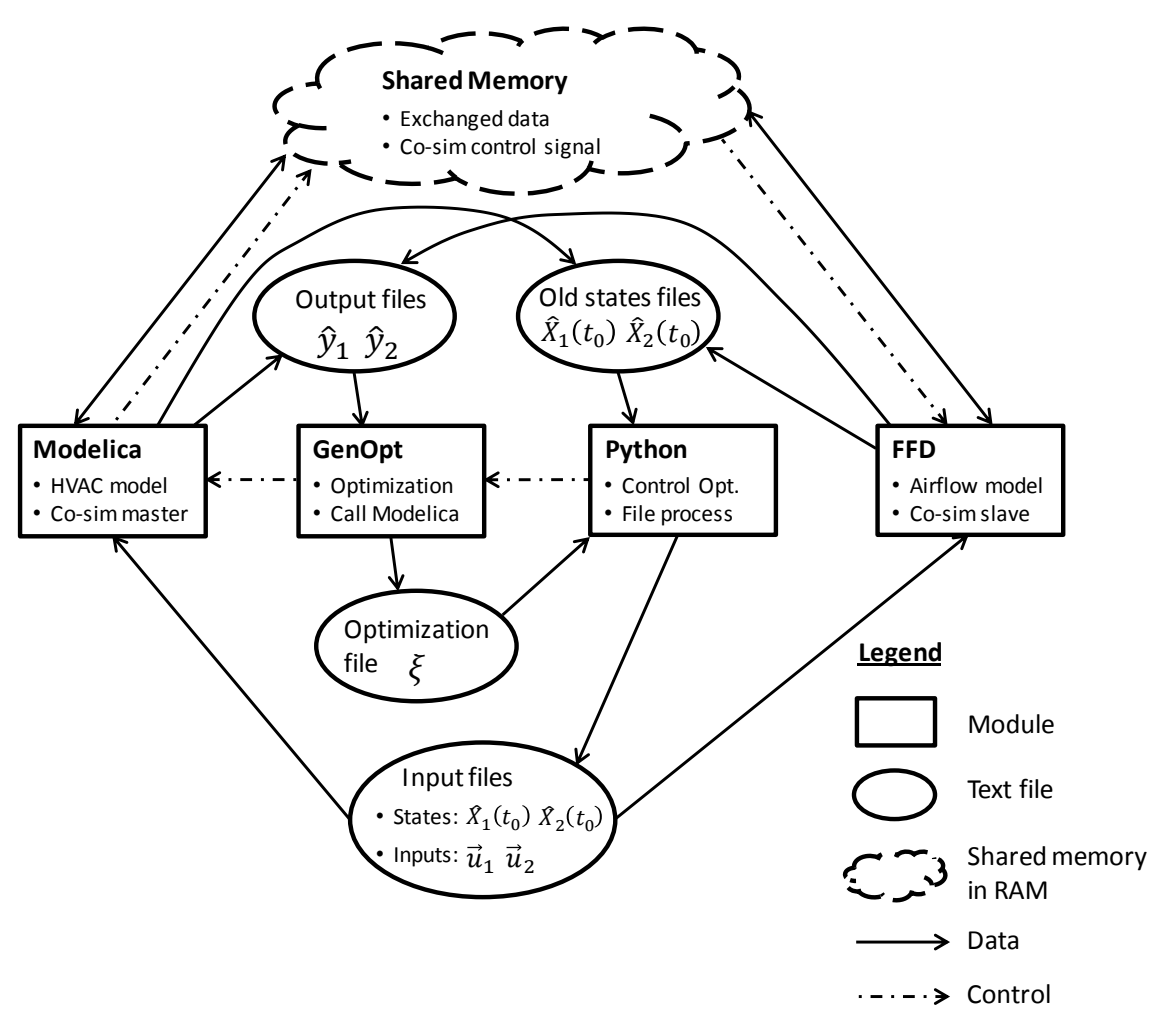

Figure 2 Implementation of the Optimization Methodology

Figure 2 shows the detailed implementation of the optimization platform based on the coupled simulation model of Modelica and FFD. The coupled simulation implementation between Modelica and FFD is largely based on the previous work (Zuo et al. 2016). Customized interfaces for Modelica and FFD are implemented to enable accessing data in the shared memory, which is recognizable to both programs. The data in the shared memory consists of exchanged data $\left(\vec{w}_{1}\right.$ and $\vec{w}_{2}$ ) and control signal that is sent from co-simulation master Modelica to control co-simulation slave FFD. In this research, we implemented a coarse grid solver in the FFD dynamic linker in Modelica Buildings library (Wetter et al. 2014) to accelerate the computation speed, which employs a plume model to correct under-predictions of the thermal plume and thermal stratification caused by the coarse grid (Jin et al. 2015). The integration of these coarse grid techniques is critical for the coupled simulation to be applied for model-based optimization, as otherwise the computational cost for FFD is too demanding when hundreds of simulations are 
needed. Before running the coupled simulation, it is required to predefine the data synchronization time step, as opposed to using adaptive time step. This is to achieve a compromise between computational cost and accuracy.

The whole implementation can be divided into:

- Reconstruct the initial values: Prior to starting the optimization, the Python module reads the text files containing states from last optimization step and initial guess of optimized variable $\vec{\xi}$, and generates text files that are to be read by Modelica and FFD to initialize their states and set up the parameters.

- Perform coupled simulations: Afterward, the Python module serving as the optimization controller fires off GenOpt, which then executes the Modelica model. To save time from compiling the Modelica source codes, one can manually compile them into an executable and request GenOpt to call it during the optimization.

- Calculate objective functions: At the end of each iteration in the optimization, Modelica and FFD exports the outputs into text files, which are then read by GenOpt to evaluate the objective functions and perform optimization to seek the optimal $\vec{\xi}$.

- Call optimization engine: GenOpt can control the optimization process until the optimal is determined. Finally, the Python module, once found that the optimization for current step is done, can move the optimization for the next step.

To further demonstrate the optimization platform, we perform a case study of seeking the optimal thermostat placement for an office room with displacement ventilation in the design phase. The objective is to achieve highest thermal comfort, or best energy efficiency, or both. In the rest of the paper, detailed validation of the coupled simulation model and optimization results will be discussed. Note that this case study is not going to conclude a general principle of thermostat 
placement since the results are case-dependent to a great extent. Instead, this case study is to demonstrate how our optimization platform facilitates model-based design for ventilation systems that involve with distinct stratified airflow and temperature distribution.

\section{Evaluation of Coupled Simulation Model}

The office room with displacement ventilation used in this study is derived from the literature (Yuan et al. 1999). The room size is $5.16 \mathrm{~m}$ by $3.65 \mathrm{~m}$ by $2.43 \mathrm{~m}$. The inflow at the displacement diffuser is $17.0^{\circ} \mathrm{C}$ with a flow rate if $183.1 \mathrm{~m}^{3} / \mathrm{h}$. Two heated dummies and two boxes are put in the room to generate the thermal plume. We hypothetically add a VAV terminal box model to the room. A pressure-dependent control logic is used in the controller. Based on the thermal load of the room, the nominal mass flow rate of the supply air at a constant temperature of $18{ }^{\circ} \mathrm{C}$ is 0.5 $\mathrm{kg} / \mathrm{s}\left(1470 \mathrm{~m}^{3} / \mathrm{h}\right)$. The thermostat is placed at the middle of the ceiling and the temperature is extracted to control VAV terminal box.

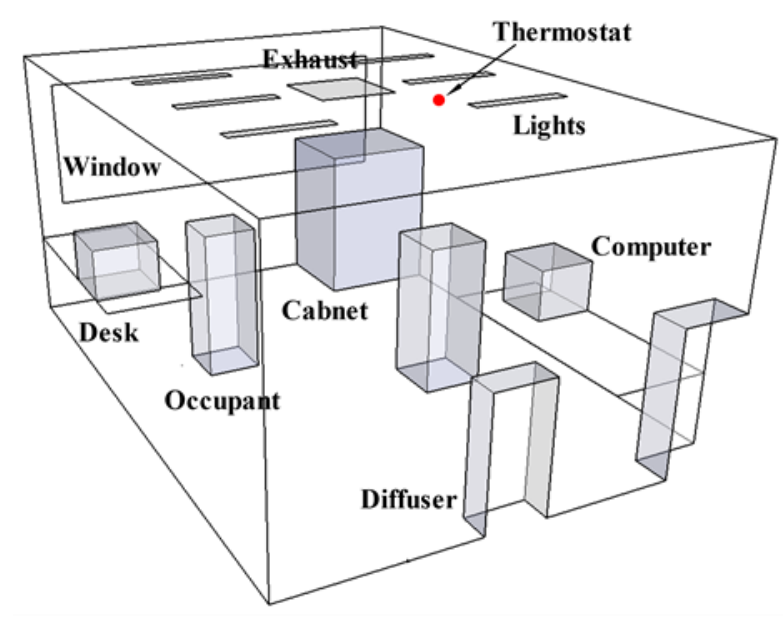

(a)

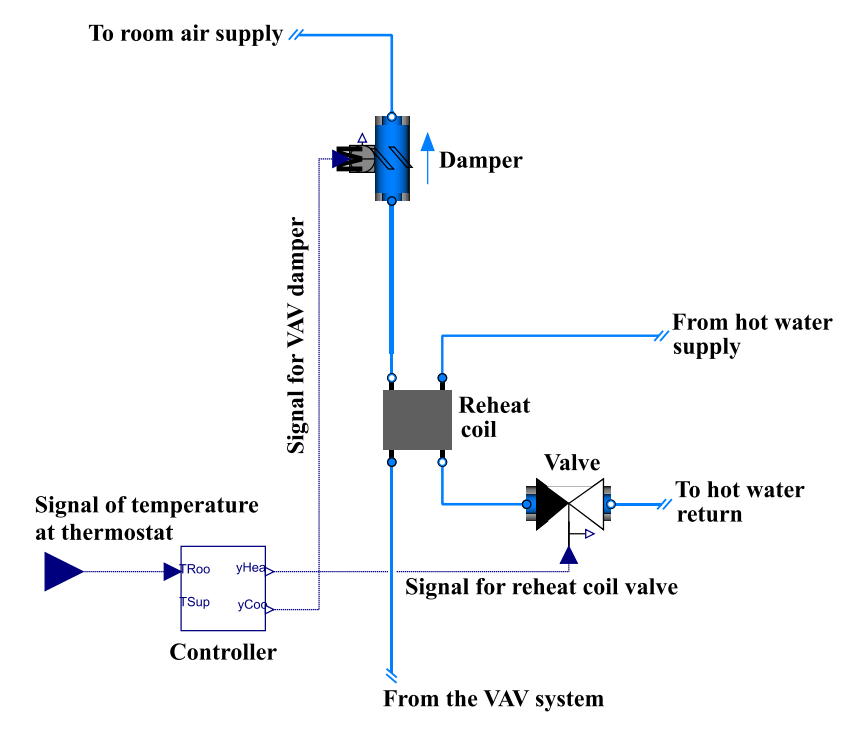

(b)

Figure 3 (a) Schematic of the office room and (b) its VAV terminal box 

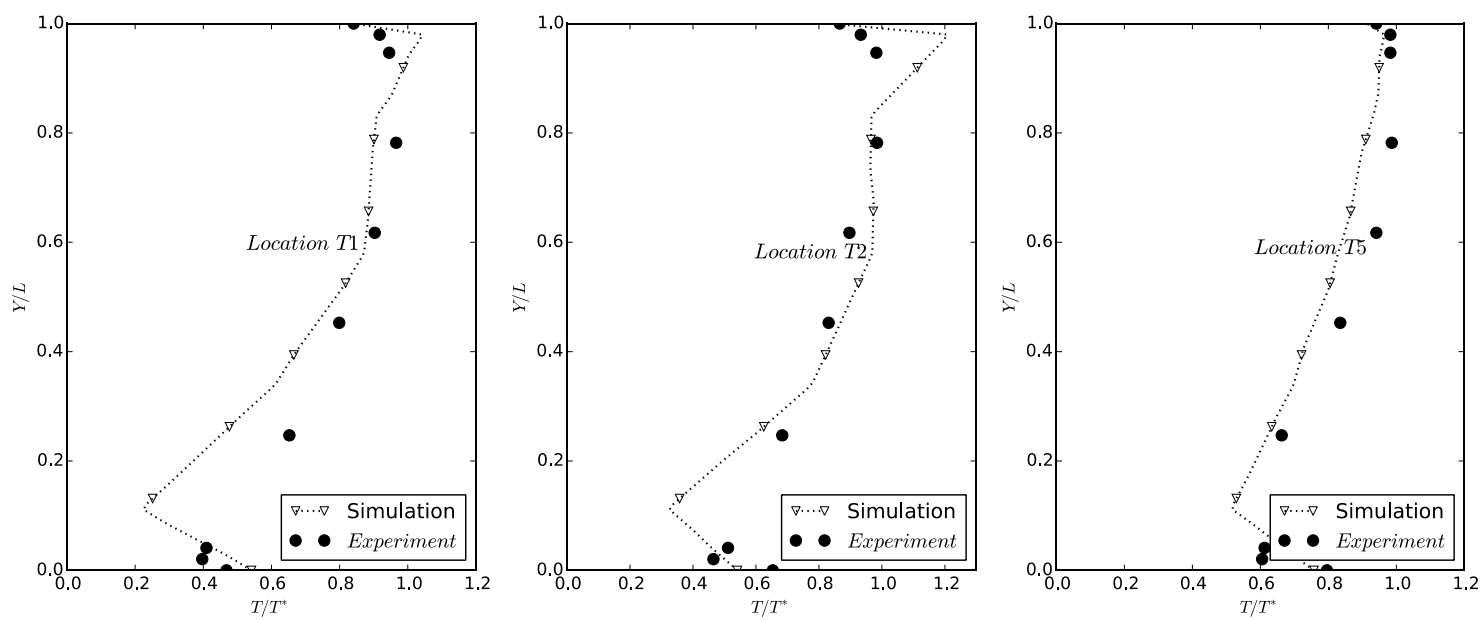

Figure 4 Temperature comparison between FFD and experimental measurements

We first validate the coupled simulation (assuming a constant supply airflow rate and temperature) against the experimental measurements. This case is directly derived from the paper by Jin et al. (2015). A coarse grid of 5 cells by 5 cells and by 8 cells is used to achieve optimal computational speed while attaining adequate accuracy. The simulation runs for 400 seconds with a time step size of 0.1 second. Figure 4 shows that the coupled simulation generally captures the variation of temperature profiles at various points in the room. We note that there are various reason why simulated results are not perfectly matching the experimental data. First, although the experiment was performed in a climate chamber where boundary conditions are well controlled, the boundary conditions can inevitably change over the course of experimental measurements. Second, the level of modeling detail is never good enough to capture all the physics regarding the flow, such as the detailed geometry of occupants and openings, the detailed heat dissipation pattern from the room items, the turbulence modeling. That is why in the paper by Jin et al. (2015), it is not surprising that even CFD with a two-equation k-epsilon model cannot generate identical results as experimental data. Nevertheless, the profiles clearly show that the temperature distribution is fairly non-uniform and stratified in vertical direction. This validation supports that the current grid together with the coarse-grid techniques in FFD can achieve a compromise between computational 
speed and accuracy. For information regarding settings in FFD, refer to Jin et al. (2015), and for information regarding setting up the coupled simulation using Modelica and FFD, refer to (Zuo et al. 2016).

We then study the transient characteristics of the VAV terminal box responding to the dynamic change of the setpoint of the thermostat. The VAV terminal box consists of a damper in air loop, reheating coil, a valve in hot water loop and a controller using a pressure-dependent control logic (Liu et al. 2012). When room temperature at sensor location is lower than setpoint (assuming a cooling season), the controller will first attempt to reduce the damper opening to decrease the supply air flowrate until a lower limit (30\% of nominal supply air flowrate) is reached. If the lower limit is reached and the room temperature is still lower than setpoint, the controller will turn on the valve in hot water loop to reheat the supply air. For the detailed model of VAV terminal box, refer to the paper by Tian, Sevilla, Zuo, et al. (2017). Here we dynamically adjust the setpoint of the thermostat in the range of 24 to $26^{\circ} \mathrm{C}$ every 10 minutes. The case is simulated for 3600 seconds ( 1 hour). Figure 5 shows the transient variation of the temperature at the thermostat, inflow temperature and flow rate, and PMV of the room. The variation of these variables is tightly related to the control logic used in this case study. Take the first spike of supply air temperature as an example. When actual temperature is lower than setpoint, the control module will first decrease the air flowrate until reaching the $30 \%$ threshold. However, the actual temperature is still lower when compared to the setpoint, then reheat using hot water will be turned on to further help increase the room temperature. When reheat is on, the supply airflow rate will be kept constant at $30 \%$ of the nominal value. After actual temperature exceeds setpoint for a certain amount of time, we can see the reheat is being shut down, and supply airflow rate climbs while the supply air temperature remains at $16^{\circ} \mathrm{C}$. We note that the temperature at the thermostat location is linearly 
interpolated using the temperatures at the neighbouring cells in FFD. This is justifiable since it has been demonstrated that the coarse grid techniques would not cause significant degradation in terms of accuracy compared to a conventional fine grid for this case (Jin et al. 2014).
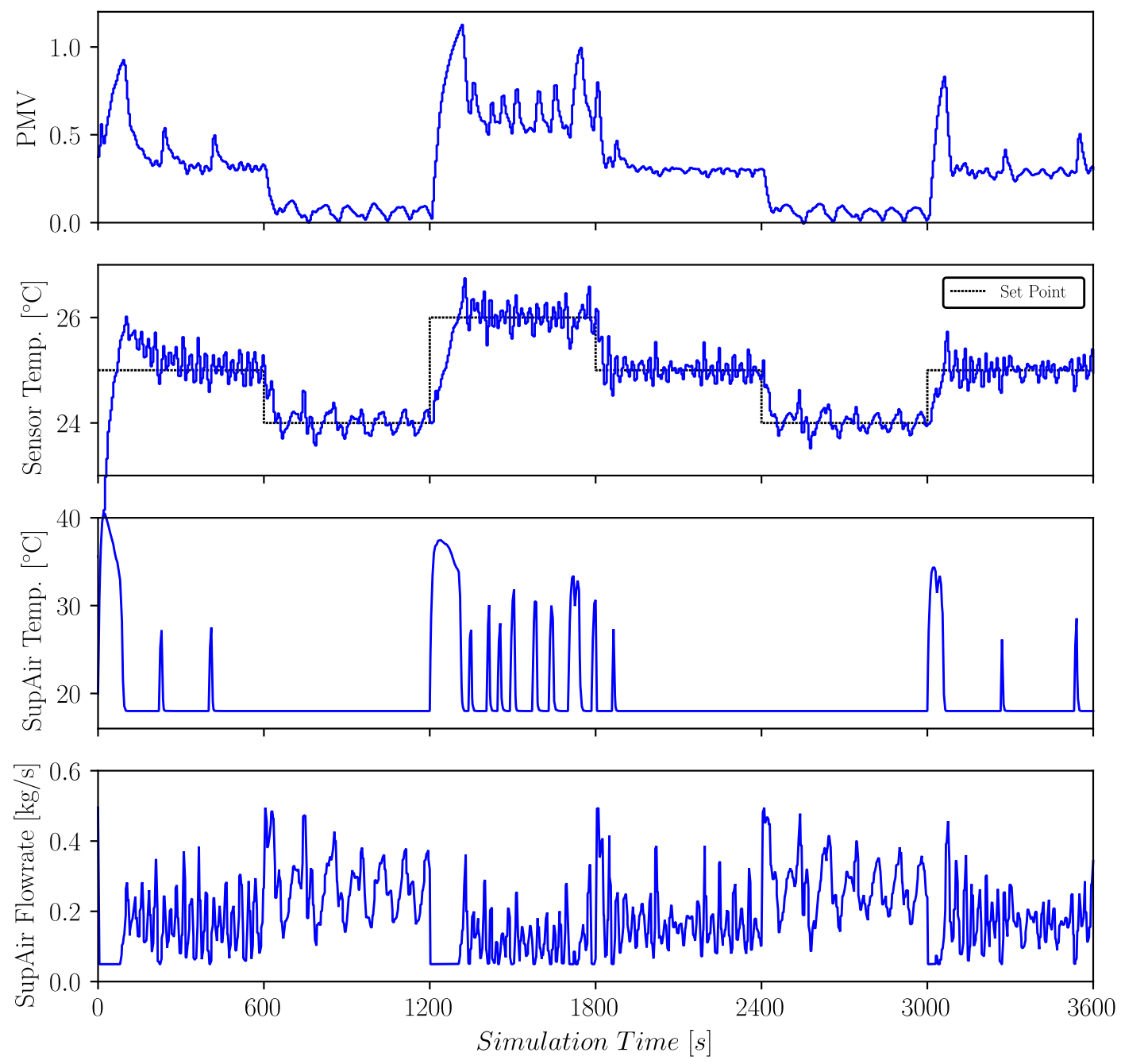

Figure 5 Dynamics of VAV terminal box and indoor environment

Due to the transient essence of the flow, the actual temperature constantly wiggles around the setpoint. Consequently, the controller module in the VAV terminal box adjusts the valves in the air loop and reheat coil to bring the actual temperature close to the setpoint. The control performance of the VAV terminal box is satisfactory as the actual temperature actively adapts to the change of 
the setpoint with a margin of $1{ }^{\circ} \mathrm{C}$. As expected, the PMV of the occupant zone, defined as the lower-half of the room, changes in a similar pattern as the actual temperature at the thermostat location. The simulation results presented in this figure also emphasise the importance of considering the dynamic control of the HVAC system using the dynamic simulation, as opposed to the steady-state simulation in previous study. The inability of the steady-state simulation to capture those dynamics may further lead to incorrect predictions of the control-performance and energy-performance of the system.

\section{Application in Optimal Thermostat Placement}

The design of the optimal thermostat placement case, which is to find the best location to place the thermostat in the design phase, is to evaluate the feasibility and performance of this platform. We will look at for an office room of typical size if this platform can predict the optimal location within a reasonably short time frame. Since a dynamic optimization process as shown in Figure 1 is essentially consisted of a series of interconnected static optimization, in which the optimization time step is equivalent to the simulation time, we configure this case study as a static optimization problem. If the static optimization can be successfully carried out in this platform, the capability of using this platform to perform dynamic optimization should be plausible. With this in mind, we first mathematically describe the optimization problem, and then we show the optimization results.

\subsection{Definition of Optimization Problem}

The objectives of the optimization study are to find the thermostat location to achieve optimal thermal comfort, or, energy efficiency, or, both, over the course of the whole simulation time. The energy consumption is estimated in terms of source energy considering both cooling and reheat. The objectives of the optimization in this case study can be formulated as: 


$$
J=\min _{\vec{\xi} \in X}\left(\vec{y}_{1} \vec{\omega}\right), \text { where } \vec{y}_{1}=(p m v, P) \text { and } \sum_{i=1}^{m} \vec{\omega}_{i}=1
$$

where $\vec{y}_{1}$ represent the output from the Modelica; $\vec{\omega}$ represents the weight for each component in $\vec{y}_{1} ; p m v$ is the predicted mean vote to quantify the thermal comfort; $P$ is the energy consumption; $\mathrm{m}$ is the number of sub-objectives; $\vec{\omega}_{i}$ is the weight for the sub-objective $\mathrm{i}$. The searching domain $\mathrm{X}$ is defined as the ceiling of the room. In this case study, the searching domain $\mathrm{X}$ covers the ceiling of the room. We note that when $p m v$ and $P$ exist in $\vec{y}_{1}$, we normalized the results to numbers with a range of $0-1$ to make them comparable in magnitude before performing the linearization.

Since the system boundary in this case study only includes the VAV terminal box (not the chilled water system), we simplify the calculation of energy consumption $P$ as:

$$
\begin{gathered}
\mathrm{P}=\int_{t_{0}}^{t_{0}+\Delta t_{\text {opt }}}\left[\eta_{1} \dot{m}_{\text {air }}\left(T_{\text {air_ret }}-T_{\text {air_sup }}\right)+\eta_{2} \dot{m}_{\text {water }}\left(T_{\text {water_ret }}-\right.\right. \\
\left.\left.T_{\text {water_sup }}\right)\right] d t,
\end{gathered}
$$

where $\eta_{1}$ and $\eta_{2}$ are conversion coefficients for chilled water and hot water, which can be found in Energy Star Portfolio Manager (EPA 2018). $T_{\text {air_ret }}$ and $T_{\text {air_sup }}$ are return and supply air temperature, respectively. $\dot{m}_{\text {air }}$ and $\dot{m}_{\text {water }}$ are mass flow rates of the air and hot water (for reheating). $T_{\text {water_ret }}$ and $T_{\text {water_sup }}$ are the return and supply water temperature. We utilize a time-average PMV to evaluate the overall thermal comfort of the occupant zone over the simulation time. It is defined as:

$$
\mathrm{pmv}=\frac{1}{\Delta t_{\text {opt }}} \int_{t_{0}}^{t_{0}+\Delta t_{o p t}} P M V_{t} d t
$$

where $P M V_{t}$ is the PMV at time $\mathrm{t}$, which is calculated by the standard thermal comfort module "Buildings.Utilities.Comfort.Fanger" in Modelica Buildings library (Wetter et al. 2014). We employed average temperature and velocity at the occupant zone predicted by CFD simulations to calculate the PMV with assuming a fixed radiative heat gain and clothing insulation. The coupled 
simulation platform makes it possible to dynamically predict the average thermal comfort at the occupant zone for a ventilation system with stratified airflow and temperature distribution. A standalone BES usually employs a well-mixed room model, which makes it difficult to estimate the thermal comfort at a certain zone or location. A standalone CFD tool should be assigned with a prescribed boundary condition, which make it impossible to simulate the dynamic interaction between indoor environment and the HVAC system.

\subsection{Optimization Results}

A typical outside condition was selected and the coupled simulation in this case study was performed for 3600 seconds ( 1 hour). The optimization time step was identical to the simulation time. A global optimization scheme called PSO was selected in GenOpt (Wetter 2009a). We performed optimizations with the settings of 10 generations and 10 particles (candidate solutions) in each generation, which are determined by some initial tests. We employed "best" neighborhood topology with a neighborhood size of 2 (Wetter 2009a). The cognitive acceleration and social acceleration are set as 2.8 and 1.3 , respectively. The variables are the coordinates of the location that the thermostat may be placed at, and the searching ranges cover the whole ceiling. Three scenarios were considered to find the optimal thermostat locations to: a) achieve best PMV (least absolute value of PMV) of the occupant zone; b) achieve least energy consumption; c) achieve an overall optimal of combined PMV and energy consumption linearized with a 50\%-to-50\% weight coefficient. Scenarios $\mathrm{a}$ and $\mathrm{b}$ are single-objective optimizations and the scenario $\mathrm{c}$ is a multiobjective optimization. Table 1 summarizes optimized results of three optimizations, in which sensor 1, sensor 2, and sensor 3 are the optimal locations corresponding to the scenario a, b and c. When thermostat is put at sensor 1 location, the thermal comfort is the highest among the three, while energy consumption is also the largest. When thermostat is put at sensor 2 location, $44 \%$ 
less energy was used compared to scenario a, while the thermal comfort is the worst. When the thermostat is placed at sensor 3 location, an overall optimal is achieved (energy consumption is lower compare to scenario a and thermal comfort is better than scenario b).

Table 1 Summary of the optimization Results

\begin{tabular}{|c|c|c|c|c|c|}
\hline $\begin{array}{c}\text { Optimization } \\
\text { Objective } J\end{array}$ & Optimal $\xi$ & $P M V$ & $\boldsymbol{E}(\boldsymbol{M} \boldsymbol{J})$ & $\begin{array}{l}\text { Number of } \\
\text { iterations }\end{array}$ & $\begin{array}{l}\text { Time cost } \\
\text { (hour) }\end{array}$ \\
\hline $\min _{\vec{\xi} \in X}(p m v)$ & $\begin{array}{c}\text { Sensor 1 } \\
(2.85,1.60,2.43)\end{array}$ & 0.14 & 33.42 & 97 & 5.8 \\
\hline$J=\min _{\vec{\xi} \in X}(P)$ & $\begin{array}{c}\text { Sensor } 2 \\
(2.55,1.79,2.43)\end{array}$ & 0.39 & 19.03 & 86 & 5.1 \\
\hline $\begin{array}{c}J=\min _{\vec{\xi} \in X}(p m v, P) \vec{\omega} \\
\overrightarrow{\boldsymbol{\omega}}=(\mathbf{5 0} \%, \mathbf{5 0} \%)\end{array}$ & $\begin{array}{c}\text { Sensor 3 } \\
(2.81,1.60,2.43)\end{array}$ & 0.15 & 29.40 & 84 & 5.2 \\
\hline
\end{tabular}

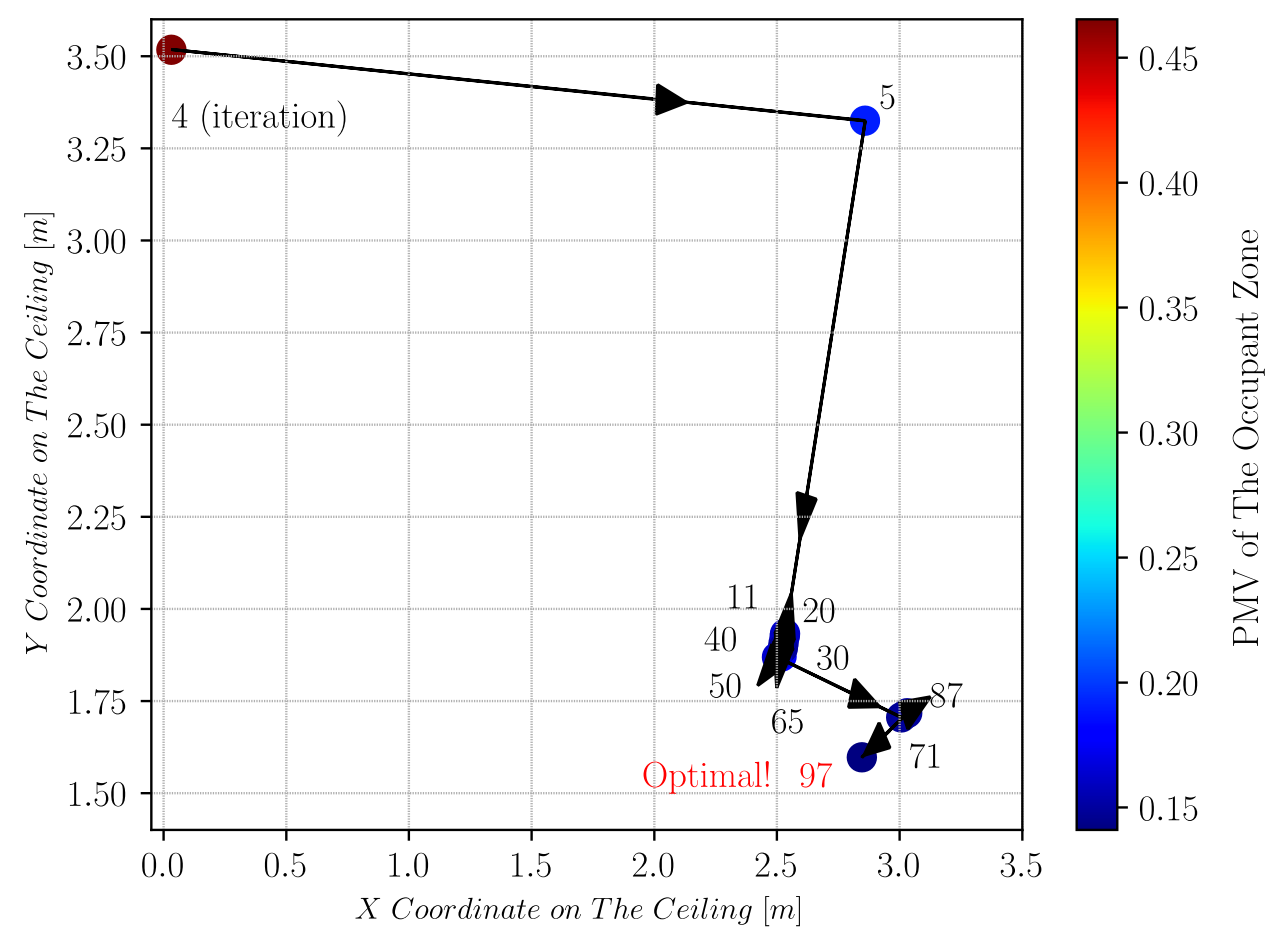

Figure 6 Search trajectory of finding optimal thermostat location leading to best PMV

The total time cost for the three optimizations is approximately 6 hours, in which up to 97 
iterations of coupled-simulation-run were performed. Figure 6 shows the searching trajectory of the optimal thermostat location on the ceiling for scenario a. Each dot in the plotting represent an iteration in the optimization process. The converging speed is plausible as: first, after approximately 5 iterations, the PMV in the optimization is close to the optimal value; after approximately 10 iterations the searching rapidly approaches the optimal location. Looking at the cluster of dots covering $10^{\text {th }}$ to $65^{\text {th }}$ iterations, we find that the PSO as a global search scheme avoids the local optimal, and eventually determines the optimal after 97 iterations. We note that the searching trajectory may depend on the initial guess. In this optimization, the initial guess of the thermostat location leads to a PMV of 0.45 , which may help accelerate the convergence speed of the optimization.

To further study the dynamics of the airflow and VAV terminal box, we present the FFD and Modelica results for the three optimization scenarios in Figure 7 and Figure 8. Over the simulation time of 1 hour, regardless of the thermostat placement, the actual temperature varies effectively with the change of the set point and the difference between them are mostly within $1{ }^{\circ} \mathrm{C}$. Neither of the three thermostat locations is significantly better or worse than others in terms of control stability and accuracy. We note that if a model based on mix-air consumption is used, the fluctuation of the actual temperature around the setpoint in the dynamic control process would be overlooked, and thus the calculation of the PMV or energy consumption might be incorrect. As expected, three different thermostat locations lead to different PMVs and energy consumption of the terminal box, due to the resulted behaviors of the terminal box in terms of cooling airflow rate/temperature, turning on/off the reheat coil. 


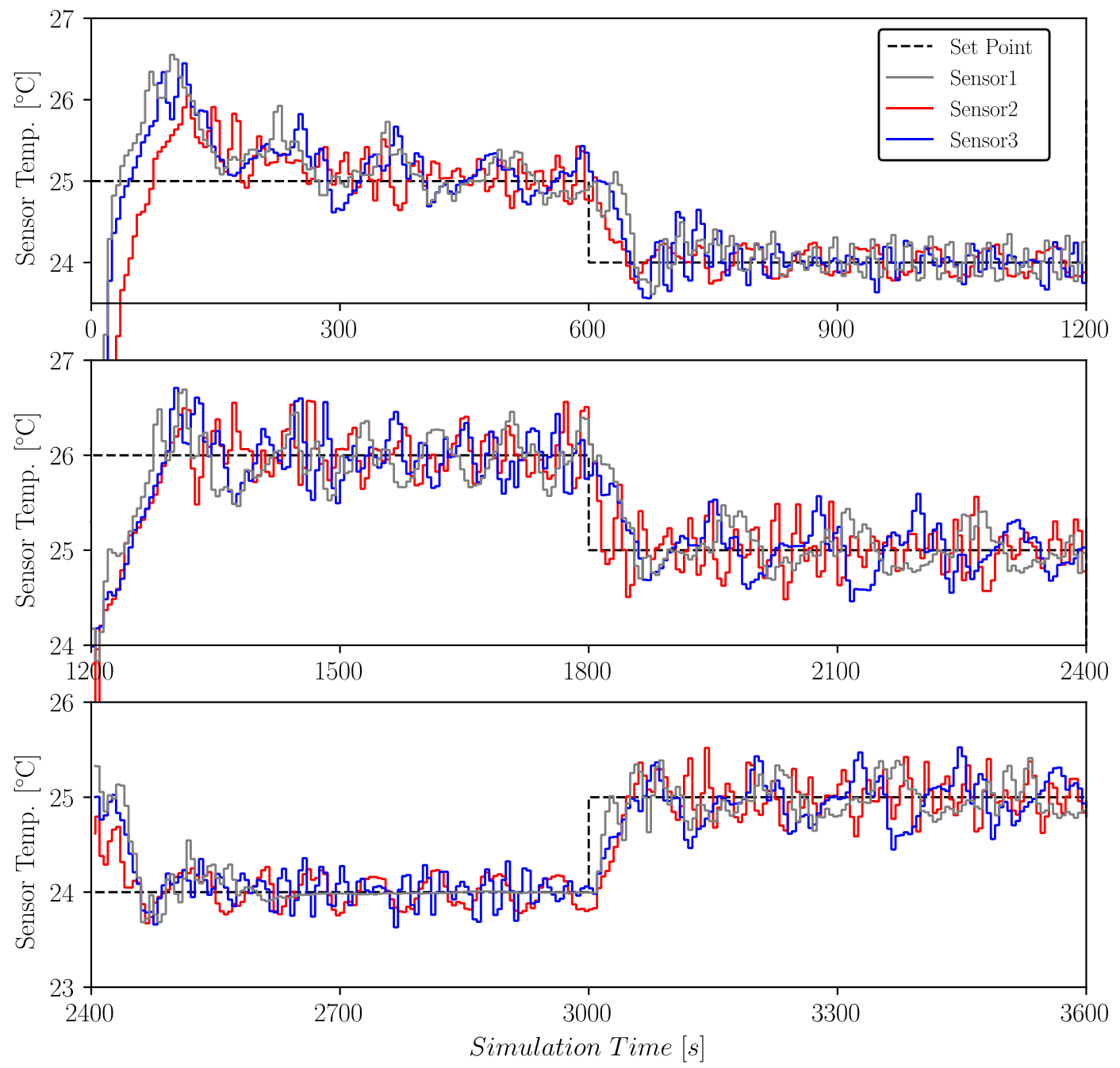

Figure 7 Time-varying temperature at the optimal thermostat locations

In Figure 8, the PMV is constantly changing with the variation of the actual temperature at the thermostat location. When thermostat is placed at Sensor 1, the time-varying PMV is closer to 0 (indicating highest thermal comfort) than the other two scenarios. This is achieved mainly by providing more cooling, as the airflow rate of the cooling air in the first scenario is the highest. Consequently, the total energy consumption, in which cooling energy accounts for the highest portion, is the largest among all. When thermostat is placed at Sensor 2, the time-varying PMV is the highest (least comfort) among all the three scenarios. This is mainly because least cooling is provided in this scenario and consequently least energy consumption is achieved, though in this 
scenario, it has the largest reheating energy consumption, which accounts very little in the total energy consumption. Lastly, when thermostat is placed at Sensor 3, an overall optimal PMV-andenergy-consumption is achieved, in which the third scenario has neither best PMV nor largest energy consumption. Its time-varying PMV is higher than the first scenario and lower than the second scenario, while its total energy-consumption is lower than the first scenario and higher than the second scenario. The reason is that the cooling provide by VAV terminal box in this scenario is in between those in scenario 1 and 2 .
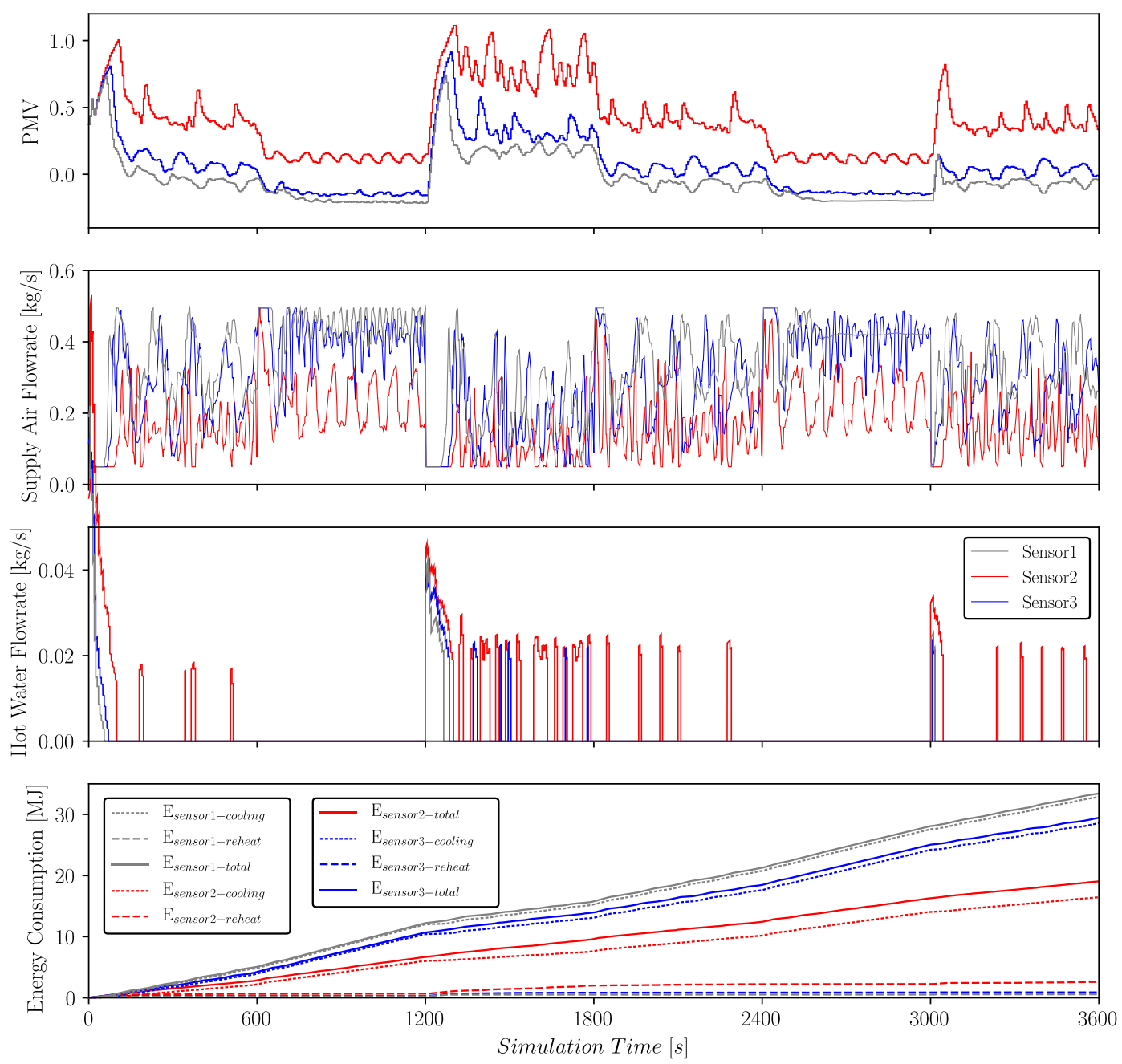

Figure 8 Responses of cooling system for optimization of PMV or energy or both

Finally, it is important to note the necessity of using a dynamic model in this kind of 
application, which involves dynamic system control. One may argue that a coupled model based on a steady-state building-energy-performance model and steady-state CFD is also viable to carry out such optimization. While this might be true, however, the omitted dynamics by the steady-state models can results into inaccurate or even incorrect results. For example, during 0 to 600 seconds in which the set point is anchored at $25^{\circ} \mathrm{C}$, a steady-state model will provide a flat/linear PMV and energy-consumption profile, as opposed to the time-varying profiles. Without comparison to the experimental measurements, we cannot conclude which one outperforms the other.

\section{Discussion}

The optimization platform has been demonstrated to seek the optimal placement of the thermostat in an office room with the displacement ventilation in the design phase. Dynamics of the VAV terminal box have been shown to be critical to determine the thermal comfort and energy efficiency. It nevertheless indicates that the proposed optimization platform can only be applied to thermostat placement or model-based design, and we have identified a few more potential applications:

1. Design optimization of HVAC system. This includes the optimization of airflow or energy system, or both. During the optimization, the interaction between the airflow and energy system will be considered, which would be critical in assessing the thermal comfort and energy efficiency. Except for the applications listed in the introduction, a good example is the design of data center cooling with raised-floor architecture, in which the open-arearatio and location of perforated tiles can be optimized to reduce cooling energy consumption.

2. Pre-commissioning (design verification) of HVAC system. Pre-commissioning or design verification is to ensure that the real system can deliver the performance as desired. Given 
the fact that the real system will dynamically change in the real operation, only verifying the design in a nominal (design) condition is not enough. Thus, the optimization platform can be utilized to optimize the settings of the cooling systems and their controls. For example, in data center cooling application, one can seek the optimal settings of a DX system to avoid short-cycling and improve control reliability.

3. Fault Detect and Diagnostics (FDD). The sensors in the thermal environment and cooling system may send back signal to indicate possible failures. It is critical, particularly in mission-critical facility, to identify whether the signal is due to the failure of the sensor or cooling system or normal fluctuation of the airflow. The optimization platform can be used to traverse all the possible failures and find the most likely one to help operators fix the fault. The highlight of this optimization platform compared to other FDD techniques lays in the fact that this optimization platform considers the interaction between the thermal environment and the cooling system.

4. Operation optimization of HVAC system. Model-based control of the cooling system in the operation would be a good application of the optimization platform. Time stepwise optimization can be performance dynamically to seek optimal settings to achieve least energy consumption or best control response. For example, in data center cooling, one can dynamically adjust the server load distribution to increase the supply air temperature or lower the supply airflow rate to save cooling energy. When the server load is projected to dramatically increase in near future, one can use the optimization platform to determine the best supply air temperature reset to precatively handle the load increase while still sustaining the energy efficiency. 


\section{Conclusion and Future Work}

In this paper, we propose an optimization framework based coupled CFD-BES simulation model. We then implement an optimization platform using a Modelica-FFD coupled simulation and GenOpt as optimization engine. The optimization platform is demonstrated to seek optimal thermostat placement in an office room with displacement ventilation and a VAV terminal box. Two single-object optimization studies show that the platform is capable to find the optimal thermostat locations to achieve best thermal comfort or energy efficiency. Due to that these two objectives are interrelated, we perform a multi-objective study by linearly combining them and find that the platform is capable to find a compromise between multiple objectives. The total time cost of the optimizations in the demonstration is about 6 hours, which are acceptable in the design phase.

We note that there are a few future studies that can be done to accelerate the speed of the coupled simulation and the optimization platform. First, parallel computing can be utilized to speed up the CFD simulations, which has been studied extensively in previous research. However, more research will be needed to couple the parallel-computing CFD simulations to BES simulations to ensure stable and efficient data transfer. Second, the technique of adaptive-synchronization-time step can be used to couple BES and CFD reduce the overhead of data transfer. Using this technique, a small-time step size is used only when it is required, i.e., to resolve step change or high-frequency dynamics. Finally, a reduced order model of indoor environment can be used to replace CFD, such that the computing speed can be drastically increased to a point, where a dynamic optimization using this platform can be carried out to assist the HVAC operations.

\section{Acknowledgment}

This research was supported by the National Science Foundation under Awards No. IIS1802017. This work also emerged from the IBPSA Project 1 , an international project conducted 
under the umbrella of the International Building Performance Simulation Association (IBPSA).

Project 1 will develop and demonstrate a BIM/GIS and Modelica Framework for building and

community energy system design and operation.

\section{Reference}

Afram, A., F. Janabi-Sharifi, A. S. Fung, and K. Raahemifar. 2017. Artificial Neural Network (Ann) Based Model Predictive Control (Mpc) and Optimization of HVAC Systems: A State of the Art Review and Case Study of a Residential HVAC System. Energy and Buildings, 141:96-113.

Blochwitz, T., M. Otter, M. Arnold, C. Bausch, H. Elmqvist, A. Junghanns, J. Mauß, M. Monteiro, T. Neidhold, and D. Neumerkel. 2011. The Functional Mockup Interface for Tool Independent Exchange of Simulation Models. Proceedings of the Proceedings of the 8th International Modelica Conference; March 20th-22nd; Technical Univeristy; Dresden; Germany.

Chen, Q. 2009. Ventilation Performance Prediction for Buildings: A Method Overview and Recent Applications. Building and Environment, 44 (4):848-58.

Chen, X., H. Yang, and W. Zhang. 2018. Simulation-Based Approach to Optimize Passively Designed Buildings: A Case Study on a Typical Architectural Form in Hot and Humid Climates. Renewable and Sustainable Energy Reviews, 82:1712-25.

D'Agostino, D., and D. Parker. 2018. A Framework for the Cost-Optimal Design of Nearly Zero Energy Buildings (Nzebs) in Representative Climates across Europe. Energy, 149:814-29.

Delgarm, N., B. Sajadi, F. Kowsary, and S. Delgarm. 2016. Multi-Objective Optimization of the Building Energy Performance: A Simulation-Based Approach by Means of Particle Swarm Optimization (Pso). Applied Energy, 170:293-303.

Du, Z., P. Xu, X. Jin, and Q. Liu. 2015. Temperature Sensor Placement Optimization for Vav Control Using CFD-Bes Co-Simulation Strategy. Building and Environment, 85:104-13.

EPA. 2018. "Portfolio Manager Technical Reference: Source Energy." https://portfoliomanager.energystar.gov/pdf/reference/Source\%20Energy.pdf.

Fu, Y., W. Zuo, M. Wetter, J. W. VanGilder, X. Han, and D. Plamondon. 2019. Equation-Based ObjectOriented Modeling and Simulation for Data Center Cooling: A Case Study. Energy and Buildings.

Han, X., J. Pei, J. Liu, and L. Xu. 2013. Multi-Objective Building Energy Consumption Prediction and Optimization for Eco-Community Planning. Energy and Buildings, 66:22-32.

Han, Y., H. Yu, and C. Sun. 2017. Simulation-Based Multiobjective Optimization of Timber-Glass Residential Buildings in Severe Cold Regions. Sustainability, 9 (12):2353.

Huang, S., W. Zuo, and M. D. Sohn. 2016. Amelioration of the Cooling Load Based Chiller Sequencing Control. Applied Energy, 168:204-15.

Huang, S., W. Zuo, and M. D. Sohn. 2017. Improved Cooling Tower Control of Legacy Chiller Plants by Optimizing the Condenser Water Set Point. Building and Environment, 111:33-46.

Jin, M., W. Liu, and Q. Chen. 2014. Accelerating Fast Fluid Dynamics with a Coarse-Grid Projection Scheme. HVAC\&R Research, 20 (8):932-43.

Jin, M., W. Liu, and Q. Chen. 2015. Simulating Buoyancy-Driven Airflow in Buildings by Coarse-Grid Fast Fluid Dynamics. Building and Environment, 85:144-52.

Lee, J. H. 2007. Optimization of Indoor Climate Conditioning with Passive and Active Methods Using Ga and CFD. Building and Environment, 42 (9):3333-40.

Li, P., H. Qiao, Y. Li, J. E. Seem, J. Winkler, and X. Li. 2014. Recent Advances in Dynamic Modeling of HVAC Equipment. Part 1: Equipment Modeling. HVAC\&R Research, 20 (1):136-49.

Liu, G., A. R. Dasu, and J. Zhang. 2012. "Review of Literature on Terminal Box Control, Occupancy Sensing Technology and Multi-Zone Demand Control Ventilation (Dcv)." In.: Pacific Northwest National Lab.(PNNL), Richland, WA (United States). 
Liu, W., R. You, J. Zhang, and Q. Chen. 2017. Development of a Fast Fluid Dynamics-Based Adjoint Method for the Inverse Design of Indoor Environments. Journal of Building Performance Simulation, 10 (3):326-43.

Liu, W., T. Zhang, Y. Xue, Z. J. Zhai, J. Wang, Y. Wei, and Q. Chen. 2015. State-of-the-Art Methods for Inverse Design of an Enclosed Environment. Building and Environment, 91:91-100.

Ma, Z., and L. Xia. 2017. Model-Based Optimization of Ground Source Heat Pump Systems. Energy Procedia, 111:12-20.

Mortezazadeh, M., and L. L. Wang. 2017. A High-Order Backward Forward Sweep Interpolating Algorithm for Semi-Lagrangian Method. International Journal for Numerical Methods in Fluids, 84 (10):58497.

Nassif, N., S. Moujaes, and M. Zaheeruddin. 2008. Self-Tuning Dynamic Models of HVAC System Components. Energy and Buildings, 40 (9):1709-20.

Nguyen, A.-T., S. Reiter, and P. Rigo. 2014. A Review on Simulation-Based Optimization Methods Applied to Building Performance Analysis. Applied Energy, 113:1043-58.

O'Shaughnessy, E., D. Cutler, K. Ardani, and R. Margolis. 2018. Solar Plus: Optimization of Distributed Solar Pv through Battery Storage and Dispatchable Load in Residential Buildings. Applied Energy, 213:11-21.

Pan, Z., Q. Guo, and H. Sun. 2017. Feasible Region Method Based Integrated Heat and Electricity Dispatch Considering Building Thermal Inertia. Applied Energy, 192:395-407.

Shehabi, A., S. Smith, D. Sartor, R. Brown, M. Herrlin, J. Koomey, E. Masanet, N. Horner, I. Azevedo, and W. Lintner. 2016. United States Data Center Energy Usage Report.

Tian, W., X. Han, W. Zuo, and M. D. Sohn. 2018. Building Energy Simulation Coupled with CFD for Indoor Environment: A Critical Review and Recent Applications. Energy and Buildings.

Tian, W., T. A. Sevilla, D. Li, W. Zuo, and M. Wetter. 2018. Fast and Self-Learning Indoor Airflow Simulation Based on in Situ Adaptive Tabulation. Journal of Building Performance Simulation, 11 (1):99-112.

Tian, W., T. A. Sevilla, and W. Zuo. 2017. A Systematic Evaluation of Accelerating Indoor Airflow Simulations Using Cross-Platform Parallel Computing. Journal of Building Performance Simulation, 10 (3):243-55.

Tian, W., T. A. Sevilla, W. Zuo, and M. D. Sohn. 2017. Coupling Fast Fluid Dynamics and Multizone Airflow Models in Modelica Buildings Library to Simulate the Dynamics of HVAC Systems. Building and Environment, 122:269-86.

Tian, Z., X. Zhang, X. Jin, X. Zhou, B. Si, and X. Shi. 2018. Towards Adoption of Building Energy Simulation and Optimization for Passive Building Design: A Survey and a Review. Energy and Buildings, 158:1306-16.

VanGilder, J. W., C. M. Healey, M. Condor, W. Tian, and Q. Menusier. 2018. A Compact Cooling-System Model for Transient Data Center Simulations. Proceedings of the 2018 17th IEEE Intersociety Conference on Thermal and Thermomechanical Phenomena in Electronic Systems (ITherm).

Wang, Q., Y. Pan, M. Zhu, Z. Huang, W. Tian, W. Zuo, X. Han, and P. Xu. 2017. A State-Space Method for Real-Time Transient Simulation of Indoor Airflow. Building and Environment, 126:184-94.

Wetter, M. 2009a. "Generic Optimization Program User Manual Version 3.0. 0." In. Berkeley, CA (United States): Lawrence Berkeley National Lab.(LBNL).

Wetter, M. 2009b. Modelica-Based Modelling and Simulation to Support Research and Development in Building Energy and Control Systems. Journal of Building Performance Simulation, 2 (2):143-61.

Wetter, M. 2011. Co-Simulation of Building Energy and Control Systems with the Building Controls Virtual Test Bed. Journal of Building Performance Simulation, 4 (3):185-203.

Wetter, M., W. Zuo, T. S. Nouidui, and X. Pang. 2014. Modelica Buildings Library. Journal of Building Performance Simulation, 7 (4):253-70.

Xue, Y., Z. J. Zhai, and Q. Chen. 2013. Inverse Prediction and Optimization of Flow Control Conditions for Confined Spaces Using a CFD-Based Genetic Algorithm. Building and Environment, 64:77-84.

Yang, C., X. Wang, M. Huang, S. Ding, and X. Ma. 2017. Design and Simulation of Gas Turbine-Based 
Cchp Combined with Solar and Compressed Air Energy Storage in a Hotel Building. Energy and Buildings, 153:412-20.

Yuan, X., Q. Chen, L. R. Glicksman, Y. Hu, and X. Yang. 1999. Measurements and Computations of Room Airflow with Displacement Ventilation. Ashrae Transactions, 105:340.

Zuo, W., and Q. Chen. 2009. Real-Time or Faster-Than-Real-Time Simulation of Airflow in Buildings. Indoor air, 19 1:33-44.

Zuo, W., and Q. Chen. 2010. Fast and Informative Flow Simulations in a Building by Using Fast Fluid Dynamics Model on Graphics Processing Unit. Building and Environment, 45 (3):747-57.

Zuo, W., M. Wetter, W. Tian, D. Li, M. Jin, and Q. Chen. 2016. Coupling Indoor Airflow, HVAC, Control and Building Envelope Heat Transfer in the Modelica Buildings Library. Journal of Building Performance Simulation, 9 (4):366-81. 